\title{
EL PRIMER REGLAMENTO DE PENSIONADOS DE LA ACADEMIA DE BELLAS ARTES EN ROMA
}

\author{
María Angeles Alonso Sánchez
}

Entre los trabajos de reorganización llevados a cabo en la Academia de San Fernando durante el período de 1754-1757 (1) merece particular atención la elaboración de los Estatutos. Realmente los aprobados en 1751 resultaban muy vagos e insuficientes, y ahora, teniendo como pauta principalmente los Estatutos de la Academia de San Lucas de Roma, se redactan unos nuevos que, aunque no exentos de defectos, habrían de tener vigencia durante muchos años (2).

Hace Caveda (3) una dura crítica de estos Estatutos, en los que - dice- «más bien parece se trataba de organizar una escuela que no una Academia", y esto por la atención que en ellos se prestaba al orden y disciplina, a las categorías de los académicos, a las obligaciones de maestros y discípulos, mientras se pasaban por alto "los trabajos científicos, la naturaleza de las discusiones» y los problemas de estética.

Pero quizá el mayor mal que los Estatutos del año 57 acarrearon a la Academia radica en el papel que se asignaba en ellos a los nobles, magnates y literatos que, con el cargo de «consiliarios" o "académicos de honor» (4), tenían entrada con voz y voto en todas las reuniones y acuerdos de la institución. Olivieri —que es de quien en principio partían las iniciativas de organización de la Academia- pretenía con esto interesar al gian público por las cuestiones artísticas, a la vez que trataba de pro-

(1) Cfr. Alonso Sánchez, M.* A.: En el centenario de la Academia de Bellas Artes de España en Roma. "Cuadernos de Prehistoria y Arqueologia", 1. U. A. M. Madrid, 1974.

(2) Dichos estatutos no fueron renovados hasta el año 1807. Cfr. Estatutos del año 1757, Leg. 3, Archivo de la Academia de San Fernando.

(3) Caveda: Memorias para la Historia de la Real Academia de Bellas Artes, tomo I, págs. $36 \mathrm{y}$ sig.

(4) Premios I. Arch. Academia de San Fernando. 
curar a los artistas un mecenazgo que estimaba había de favorecer el desarrollo del arte. Sin embargo, no fue así, sino que con tal injerencia "condenaba al artista a una tutela infructuosa" (5), a la vez que daba lugar a que un conjunto de señores más o menos eruditos, pero en su mayor parte desprovistos de conocimientos artísticos, fueran los que con su parecer decidieran el rumbo a seguir en una serie de cuestiones totalmente ajenas a su competencia. Y en esto ciertamente no habían copiado los españoles a la Academia de San Lucas, pues allí, según se preceptuaba en los Estatutos, estaban excluidas de las Juntas las personas no facultativas, teniendo entrada estos consiliarios y académicos de honor sólamente a las solemnes distribuciones de premios del Capitolio (6).

De todos modos, no puede negarse que el amor al arte, el interés por las cuestiones arqueológicas, el deseo de superación y de gloria se hizo patrimonio de nobles y literatos. Expresión de este espíritu son los discursos y composiciones poéticas leídos en las Juntas públicas y en las solemnes distribuciones de premios (7). Y fruto de tal interés fueron también los reglamentos de pensionados en el extranjero.

El apartado que, bajo el título "Discípulos de Roma», aparecía en la legislación de 1751 se había convertido ahora en dos artículos, el XX y el XXI, titulados, respectivamente, "Pensionados en Roma» y «Director de los pensionados en Roma» (8). Seguía vigente en ellos el número de seis pensionados determinados en los tiempos de la Junta Preparatoria, pero se añadían algunas innovaciones de capital interés: la primera la creación del cargo de director de pensionados -igual que tenían las Academias de otras naciones-, al que deberían aquéllos sujetarse en el terreno disciplinar y artístico. Tarea del director había de ser no sólo controlar y guiar los estudios de los pensionados, dando puntual cuenta de todo a la Academia, sino también velar sobre la conducta de aquéllos, servir de eslabón entre éstos y el embajador o ministro del Rey en Roma y procurarles entrada en museos y escuelas a fin de que el aprovechamiento fuera máximo.

Una innovación se introducía también con la determinación del tiempo (seis años) que los pensionados habían de residir en Roma, pasado el cual se obligaban a restituirse al Reino. Y otra en el terreno financie-

(5) Caveda: Op. cit., pág. 36.

(6) Busiri-Vici: Sessantacinque anni della Accademia di S. Luca. «Ordini e statuti dell'Academia del disegno dei pittori, scultori e architetti di Roma, sotto il titolo e patrocinio di S. Luca, sotto gli auspizi del Santissimo Padre Clemente XI.»

(7) Libros de Premios de la Academia de San Fernando. Años 1753-1780. Arch. de la Academia.

(8) Estatutos de 1757. Leg. 3. Arch. Academia de San Fernando. 
ro, ya que desde este momento pasaban a depender económicamente de la Academia. Podía ésta costearles las pensiones sin gran esfuerzo, pues desde el año 1752 había sido dotada espléndidamente por Fernando VI con la suma anual de 12.500 pesos (9).

Aprobó el Rey los Estatutos presentados por la Academia el 30 de mayo de 1757 y los académicos y consiliarios descansaron de sus tareas de organización durante el verano. Pasados «los rigores de la estación estiva" volvieron de nuevo al trabajo para elaborar una "Instrucción» por la que habían de regirse los pensionados de Roma.

El año 1758 se empleó casi por entero en estudiar y discutir los puntos que debería abarcar el Reglamento, tarea no fácil, pues eran diversísimas las opiniones de consiliarios, directores y académicos (10). $\mathrm{Al}$ fin, en la Junta Particular del 28 de septiembre (11) recibía aprobación (12), teniendo vigencia este Reglamento durante todo el siglo XVIII, pues aunque más de una vez en el transcurso de los años se pidieron nuevos informes con intención de elaborar otra Instrucción, nunca llegó a realizarse tal intento.

Tenía el Reglamento (13) una primera parte compuesta por diez artículos en los que se señalaban las obligaciones del director y de los pensionados en general. Solamente aparecía aquí una innovación con respecto a lo ya establecido en los artículos 20 y 21 de los Estatutos de la Academia, de los que eran casi una glosa. Esta innovación era la prohibición de que los pensionados se casasen «sin pedir primero y obtener licencia de la Academia" (14). Pensaban, sin duda, los académicos que el casarse en Italia podría ser un grave inconveniente para que los pensionados se restituyeran al Reino una vez cumplido el tiempo de sus pensiones, como de hecho lo había sido para Francisco Preciado.

El director quedaba dependiendo directamente de la Academia, a la que debía recurrir en las dudas que en el ejercicio de su cargo le sobreviniesen, si bien se prevenía el caso en que por necesitar una pronta res-

(9) Caveda: Op. cit., pág. 33.

(10) Dictámenes de profesores. Año 1758. Leg. 50. Arch. Academia de San Fernando.

(11) Juntas Particulares, año 1758. Arch. Academia de San Fernando.

(12) La parte relativa a los arquitectos no estaba aún elaborada en esta fecha y se aprobó en la junta del día 20 de noviembre.

(13) Instrucción para el director y pensionados de Roma. Leg. 50. Archivo de la Academia de San Fernando.

(14) Instrucción para el director y pensionados de Roma. Art. 8. Leg. 50. Archivo Academia de San Fernando. 
puesta no pudiese acudir a la misma, ordenándosele que en tales circunstancias acudiese al agente general del Rey (15).

A continuación se extendían los artículos concernientes a cada una de las ramas de pensionados, pintores, escultores y arquitectos, dándose normas abundantes y concretas para cada uno de ellos.

\section{PENSIONADOS POR LA PINTURA}

Para la redacción de los artículos concernientes a los pensionados de pintura, la Academia encargó a los profesores Antonio González Ruiz, Pablo Pernicharo y Antonio González Velázquez que extendieran unos informes en los que se determinara el empleo que los pensionados de este arte deberían hacer de los seis años que duraba su pensión. Estos tres profesores eran en esa fecha directores de la Academia y unían a esto la experiencia de haber vivido algunos años en Roma, ya a sus expensas (16), ya como pensionados (17).

Extendieron sus informes los profesores sin quebrarse mucho la cabeza. Son dictámenes bastante imprecisos y no forman un plan de estudios, sino más bien un conjunto de consejos más o menos acertados. Coinciden los tres en recomendar que durante los primeros años copien los pensoinados obras antiguas ("estatuas célebres», dice solamente Antonio González Ruiz), que después copien a Rafael y a Miguel Angel en las estancias y Capilla Sixtina (18) y a Aníbal Carracci y al Dominiquino (19), y que en los últimos años hagan obras de "su caudal». Deseaba Pernicharo que el director les facilitara la entrada en los palacios donde están los mejores cuadros y advertía que "por ningún modo se les permita que determinadamente se empeñen en seguir una manera que suele ser motivo de su ruina, sino mirando sólo al principal objeto, que es copiar del natural» (20). También González Ruiz se mostraba partidario de que los pensionados copiasen del natural e incluso llegaba a sugerir que se les destinase un hombre como modelo y se les organizase una Academia de noche. Pero a esto, como era de suponer, la Academia no accedió, pues pareció a todos más conveniente que los pensionados asistieran a

(15) Ibid. arts. 4 y 5 .

(16) Antonio González Ruiz.

(17) Pernicharo había sido pensionado por Felipe V y Antonio González Velázquez por la Academia de San Fernando.

(18) Dictamen de Antonio González Velázquez. Leg. 50. Arch. Academia de San Fernando.

(19) Dictamen de Antonio González Ruiz. Leg. 50. Arch. Academia de San Fernando.

(20) Dictamen de Pablo Pernichero. Leg. 50. Arch. Academia de San Fernando. 
las clases de desnudo organizadas por Benedicto XIV en el Capitolio (21). Es extraño que no se le ocurriera esta solución a Antonio González. Quizá participaba de la opinión de aquel sector de romanos que habían opuesto a la Academia del Capitolio duras críticas por estar en un lugar muy apartado, en calles llenas de mal vivientes y en una zona alta, azotada por los vientos, lo que hacía difícil el acceso en los días de invierno (22).

Los tres profesores señalaban una serie de dibujos y pinturas que los pensionados deberían enviar a la Academia, dibujos y pinturas con los cuales la Academia podría controlar el progreso de sus enviados, pero sobre todo podría enriquecer el naciente Museo de San Fernando y servirían de modelo a los discípulos de Madrid. Pernicharo, el menos exigente de los tres, pedía solamente que al final de su pensión enviara cada pensionado dos cuadros, uno copia de un buen autor y otro de invención propia. En cambio, Antonio González Ruiz establecía una serie de dibujos para los dos primeros años (23) y una copia durante cada uno de los años siguientes. Y Antonio G. Velázquez pedía que cada año enviasen "alguna obra» a la Academia, sin especificar mucho qué tipo de obra.

Es curiosa la advertencia de este director de que los pensionados «huyan de la escuela de Jordán y Chicho Solimena (24) por la larga experiencia que hay de haberse perdido los que siguieron estos principios", advertencia que fue recogida en la Instrucción, quedando dispuesto que los pensionados sigan siempre «la escuela romana, lombarda o veneciana» (25).

A base de estos tres informes (y teniendo muy presente el dictamen dado por Felipe de Castro para los escultores) se elaboró la Instrucción. Abarca ésta trece artículos y en ella Ignacio de Hermosilla (que fue quien la redactó como secretario de la Academia) subsanó con creces lo que de impreciso tenían los dictámenes de los profesores, dejando muy determinados los trabajos que cada pensionado había de realizar en cada uno de los seis años y también las pruebas de su arte que había de enviar a la Academia.

(21) Artículo 1.

(22) Busiri-Vici: 65 anni della Accademia di S. Luca, pág. 84.

(23) Doce cada año, hechos en papel blanco con lápiz encarnado o negro. Dictamen de Antonio González Ruiz. Leg. 50. Arch. Academia de San Fernando.

(24) Francisco Solimena, llamado comúnmente «L'Abbate Ciccio».

(25) Art. 13: "Instrucción para el Director y los Pensionados del Rey en Roma». 
Y así se preceptúa que en el primer año (26) se limiten a dibujar (dejando el problema del color para el año siguiente) estatuas antiguas (27) y cuadros de buenos autores de la escuela romana, lombarda o veneciana (28), sometiéndose siempre a la orientación y corrección del director. Como prueba de su aprovechamiento enviarán cada uno al final de este primer año «seis dibujos (dos de pinturas y cuatro de las estatuas), todos de un mismo tamaño, que será de un pliego de papel Holanda de marca» (29). Además se les ordena que envíen cada uno dos docenas de las mejores academias que trabajen, a juicio del director.

Durante el segundo año (30), sin dejar de practicar lo hecho en el primero, se aplicarán ya a los colores, copiando alguna obra de alguno de los pintores señalados para el primer año y enviando a la Academia al final de éste tres copias de pinturas de Rafael, en lienzos de cinco pies de alto por cuatro de ancho (o al contrario), además de cuatro dibujos como los del año precedente.

En el tercer año (31) proseguirán los pensionados dibujando por el natural y copiando cuadros de los grandes autores antes citados. Y al final del año enviarán cada uno seis copias en lienzos de iguales medidas que los originales.

Para el cuarto año (32), además de continuar copiando (debían enviar a la Academia cuatro copias en lienzos iguales a los originales), se determina que comiencen a hacer cuadros de invención propia y que envíen uno a la de San Fernando.

Dos cuadros de invención propia, pero con tema enviado por la Academia, se preceptúan para el quinto año (33), además de cuatro copias en las mismas condiciones que el año anterior. $\mathrm{Y}$ dos cuadros de invención, de tamaño de dos varas y media de alto por dos de ancho, y con tema dado por la Academia, se preceptúan también para el

(26) Ibíd. Leg. 50. Arts. 12, 13 y 14.

(27) «El Hércules Farnese, el Antinoo, el Apolo, el Laoconte v el torso de Belvedere, el Apolo de Médicis; las dos Venus, y otras así desnudas como vestidas". (Esta relación fue tomada del Dictamen hecho por Felipe de Castro para los escultores.)

(28) "Obras de Rafael, Anival, Dominiquino, Guido, Lafranco, Saqui, Cortona, Marati, Cerezo, Parmesano, Veronés, Ticiano (sic) o de otros célebres pintores». Art. 13. Instrucción citada.

(29) Instrucción citada. Art. 14.

(30) Instrucción citada. Arts. 15 y 16.

(31) Instrucción citada. Art. 17.

(32) Instrucción citada. Arts. 18 y 19.

(33) Instrucción citada. Art. 20. 
sexto año (34), además de las consabidas copias (dos en este año) de los pintores célebres.

Se trataba, pues, de una cantidad considerable de dibujos y cuadros. Y es de notar que en el artículo 23 , último de los concernientes a pintores, se determinaba que la Academia habría de costear todos los lienzos, colores y bastidores de las referidas obras y de cuantas los pensionados quisieran voluntariamente enviar.

\section{PENSIONADOS POR LA ESCULTURA}

La Academia pidió a los profesores de escultura Juan Domingo Olivieri, Pascual de Mena y Felipe de Castro que enviaran sus dictámenes. De los tres gustó especialmente a los académicos el presentado por este último, pues era el más completo y ofrecía todo un plan organizado de trabajos, visitas artísticas y consejos llenos de experiencia. En cuanto a los informes presentados por los otros dos profeores, en el elaborado por Mena se limitaba éste a sugerir que los escultores se dedicasen a copiar estatuas antiguas, enviando cada año a la Academia alguna prueba de su aplicación. Y Olivieri, que comenzaba su dictmen con una protesta por el poco fruto que los pensionados de Roma habían dado a su Patria (35), tenía un plan en el que se notaba que pesaba más su afán de enriquecer a poco precio con moldes y estatuas la Academia de San Fernando - que tan en el corazón llevaba-, que no el bien de los pensionados. Pretendía que éstos se ocupasen el primer año en dibujar y modelar "del antiguo». Y durante los cinco restantes dedicasen, alternativamente, uno a copiar estatuas antiguas y otro a realizarlas de su invención "para que la Academia se halle en poco tiempo y a poca costa con buenas copias de lo antiguo y, al mismo tiempo, con buenos originales de artífices españoles». E incluso presenta un presupuesto de lo que esto vendría a costar a la Academia para demostrar las ventajas económicas que en su plan había (36).

Pero prevaleció, como era de esperar, el dictamen de Felipe de Castro, y a base de él, casi exclusivamente, elaboró el secretario la Ins-

(34) Instrucción citada. Art. 21.

(35) «He reparado cómo los pensionistas de Roma, en los muchos años que han permanecido en aquella Corte, por muy notorios que hayan sido sus adelantamientos, no han dado ni a su Rey ni a su Patria ningún fruto que eternice la gloria de sus nombres.» Dictamen de J. D. Olivieri. Leg. 50. Archivo Academia de San Fernando.

(36) Dictamen de Juan D. Olivieri. Leg. 50. Arch. Academia de San Fernando. 
trucción. Tenía de común con la realizada para los pintores la obligación que se establecía de que los escultores asistieran a la Academia de Desnudo del Capitolio (37) y también la relación de las estatuas antiguas que los pensionados deberían dibujar en el primer año (38). Pero se ordenaba que cada pensionado escogiera un maestro estatuario al que consultara sus dudas y bajo cuya dirección trabajase (39), si bien deberían guardarse mucho los discípulos de copiar sus obras, debiendo, en cambio, dirigir la atención a aquellas antiguas y a otras modernas de autores ya consagrados (40).

Se aconseja que lleven siempre consigo "libros de memoria", donde apunten las obras más dignas que encuentren en los templos, palacios, jardines, fuentes, etc. También se ordena que el director lleve a los pensionados a las casas de los artífices que esculpen en piedras orientales para que observen cómo se elaboran las medallas de oro, bronce y plata y cómo se hacen los cuños de acero y los moldes y fundiciones para las estatuas de bronce (41).

La instrucción dedica seis artículos a concretar las obras que los pensionados deberían enviar a la Academia y el plan es aquí mucho más ambicioso de lo sugerido por los profesores, pues establece para el primer año una copia en barro de tres cuartas castellanas de alto, que cada uno ha de enviar después de hacerla cocer. En el segundo, dos de estas copias de una vara de alto. En el tercero, una copia en estas mismas condiciones y otra en barro del mismo tamaño que el original. En el cuarto año, una estatua de invención propia, en tamaño natural, que habría de representar a la persona que previamente indicase la Academia. En cada uno de los años quinto y sexto, una copia en mármol del tamaño del original y un bajo relieve de su invención, si bien ajustándose al tamaño y asunto que la Academia determine (42).

Del mismo modo que se había fijado para los pintores, la Academia se comprometía a costear los materiales e instrumentos precisos. Y recogía la iniciativa de Felipe de Castro de que se sacasen moldes de

(37) Art. 27. Instrucción para el Director y los pensionistas del Rey en Roma.

(38) Art. 25. Ibídem.

(39) En su dictamen nombraba Felipe de Castro a los tres escultores que, a su juicio, verdaderamente merecían la categoría de maestros en Roma. Eran éstos: Felipe del Valle, Pedro Bracci y Francisco Vergara.

(40) «Sea precisamente de Micael Angelo, del Bernino, de Algardi, del Flamenco, de Hercole Ferrata, de Antonio Raggi, Melchior Cafa, Camilo Rusconi, Angelo Rosi, Pietro le Gros u otros semejantes.» Art. 26. Instruccióun citada.

(41) Art. 29. Ibídem.

(42) Arts. 32-37. Ibidem. 
las estatuas de mármol y se dejase un vaciado de ellas en Roma para el estudio de los pensionalos, enviando a la Academia la estatua y el molde a fin de que ésta pudiese fácilmente proveerse de vaciados para la enseñanza.

\section{PENSIONADOS POR LA ARQUitectura}

Poseemos los informes presentados por Ventura Rodríguez, José de Hermosilla, Jaime Marquet y el Conde de Aranda, que fueron la materia prima de la que Ignacio de Hermosilla sacó el Reglamento. De todos ellos el más extenso y detallado es el de José de Hermosilla y de éste fue, por disposición de la Junta (43), del que más normas se adoptaron. El de Ventura Rodríguez pecaba de vago e impreciso; el de Marquet se limitaba a exponer la conveniencia de que los pensionados no pasasen los seis años en Roma, y el del Conde de Aranda era un modelo acabado de disertación erudita y pesada, sin aportaciones que realmente valieran la pena. Sin embargo, prevalecieron las ideas de éste en cuanto al orden de lugares que debían rcorrer los pensionados en los seis años de pensión. Era éste un punto de gran discordia, pues mientras Ventura Rodríguez aprobaba el que - al igual que los demás pensionados- permanecieran los arquitectos todo el tiempo de su pensión en Roma, Jaime Marquet opinaba que para su mayor adelanto deberían ir por primera providencia a París y permanecer allí cuatro años para tomar un conocimiento de las «distibuciones de las casas", y luego pasar a Roma durante los dos últimos "para estudiar, sobre todo, las proporciones de los edificios antiguos que son en aquella Corte y sus cercanías» (44). Tampoco parecía bien a Hermosilla que permanecieran los seis años en Roma, pues juzgaba que, dado que en Roma no había estudio público de arquitectura, convendría más que la preparación teórica la hiciesen en España (45), y aun empezasen su viaje "por El Escorial y demás palacios y obras insignes de España, gastando en ellos dos años». Después podrían los pensionados invertir otros dos años en recorrer Francia, Flandes y aun Londres, y finalmente dedicar los dos restantes a Roma e Italia.

(43) Junta particular del día 28 de septiembre. Año 1758. Arch. Academia de San Fernando.

(44) Dictamen de Jaime Marquet. Leg. 50. Arch. Academia de San Fernando.

(45) Dictamen de José de Hermosilla. Leg. 50. Arch. Academia de San Fernando. 
Prevaleció, sin embargo, la opinión del Conde de Aranda y así se determinó que los pensionados dedicasen los dos primeros años de su pensión a estudiar y conocer Roma, pasando a conocer durante los dos años siguientes las principales ciudades de Italia (la Instrucción señala concretamente Bolonia, Florencia, Milán, Génova y Venecia), Alemania y Flandes, destinando los dos últimos años a Holanda, Londres y Francia (46).

El plan estaba, pues, concebido en gran escala. Sin embargo, no me consta que en vida de Preciado se llegaran a realizar estos viajes por Italia y las otras naciones europeas.

En cuanto a las asignaturas y estudios que deberían cursar, la Academia aceptó plenamente el plan presentado por Hermosilla, que era el más extenso, limitándose casi a reducir a artículos el memorial de aquél. Y así el artículo primero fijaba que en los dos primreos años, durante su permanencia en Roma, asistieran los discpulos «de cuando en cuando» a las cátedras de matemáticas para renovar y fijar en la memoria las nociones de la «Aritmética, Geometría, Estática, Hidrostática, Hidrometría, Maquinaria, Perspectiva, Trigonometría, Seociones cónicas y Montea" (47).

Salía la Academia al paso de la dificultad presentada por Hermosilla (48), estableciendo en el artículo 3 que aún cuando en Roma no existía un estudio público de arquitectura, sería tarea del director poner a los pensionados en comunicación con los profesores más doctos, a fin de que «tratando y conferenciando con ellos hagan con más luz y provecho sus estudios» (49).

Se daba importancia capital al estudio de Vitrubio (50), encarecido singularmente por Hermosilla. Precisamente había hecho el propio Hermosilla una nueva traducción del autor latino, con numerosas anotaciones y explicaciones de los pasajes difíciles, traducción que desgraciadamente no se llegó a publicar, pero cuya elaboración había dado al traductor un profundo conocimiento de Vitrubio (51). Y para que el estudio fuera más provechoso se aconsejaba recurrir a sus comentadores, singularmente «al señor Patriarca de Aquileya, Daniel Bárbaro; Guillermo

(46) Instrucción para el Director y pensionados de Roma. Leg. 50. Arch. Academia de San Fernando.

(47) Ibidem.

(48) «... hablo de la experiencia: No hay estudio público de arquitectura en Roma». Dictamen de Hermosilla. Fol. 4. Leg. 50. Arch. Academia de San Fernando.

(49) Instrucción citada. Art. 3.

(50) Instrucción citada. Art. 4.

(51) Libro de Premios. Año 1760. Arch. Academia de San Fernando. 
Philandro, León Bautista Alberti y otros", sin olvidar el compendio escrito en francés por Ms. Perrault (52).

Obligación de los pensionados era completar este estudio con la lectura de los más famosos autores, tales como "Serlio, Paladio, Scamozi, Vignola, Juan Arfe, Villafañe, el Padre Fr. Lorenzo de San Nicolás, el señor Obispo Caramuel, el Padre Vicente Tosca y otros» (53).

Una vez en posesión de estos conocimientos teóricos, debía procederse al estudio y reconocimiento de los edificios y proyectos estampados en numerosos libros a fin de meditar y observar, a la luz de los estudios precedentes, "en qué se ajustan o apartan de los preceptos». Pasarían los pensionados después a la contemplación y estudio directo de los edificios antiguos famosos, "enteros o medio arruinados", que han quedado en aquella Corte, ejercitándose en «notar sus situaciones, indagar la construcción de sus fundamentos, las precauciones con que están hechos, los cortes de sus piedras, las proporciones de los vanos con los macizos, los adornos que les han quedado, etc., dieñándolo todo con la mayor exactitud" (54).

El mismo estudio habría de hacerse sobre los edificios modernos sagrados y profanos, dirigiendo la atención especialmente a las comodidades que dichos edificios presentasen, a la distribución de sus dependencias, a la calidad de los materiales empleados y a las máquinas usadas en su construcción (55).

Sólo después de estos dos años de intenso trabajo y estudio, durante los cuales debían los pensionados enviar determinadas pruebas de su aplicación, habían de hacer y remitir a la Academia «diseños de invención propia».

(52) Bárbaro, Daniel: I dieci libri dell'parchitettura. Venecia, 1556; Philandro, Guillermo: In decem libros M. Vitrubii Pollionis de Architectura Annotationes... Roma, 1544; Alberti, León Bautista: L'Architettura, Tradocto in lingua florentina da Cosimo Bartoli. Firenze, 1550. Perrault, Claude: Abregé des dix livres d'Architecture. París, 1674.

(53) Sebastián, Serlio: Regole generali di Architettura. 3.' ed. Venecia, 1562; Paladio, Andrés: Libro primero de su Arquitectura, que trata de cinco órdenes para fabricar, traducido del toscano al castellano por Francisco de Praves. Valladolid, 1625; Scamozi, Vicenzo: Discorsi sopra l'antichita di Roma, 1582; Vignola: Regla de las cinco órdenes de arquitectura de Jacome de Vignola. Ahora de nuevo traducido de Toscano en Romance por Patricio Caxessi. Madrid. En casa de Vicencio Carduccho, 1593; Arfe, Juan: De varia commesuración para la escultura y architectura. Sevilla, 1585; Fr. Lorenzo de San Nicolás: Del arte y uso de la arquitectura. Madrid, 1663; Caramuel, Juan Obispo: Cursus mathematicus: arithmetica, algebra et geometria. Companiae, 1667; Tosca, Vicente P.: Compendio Mathematico. Madrid, 1727.

(54) Instrucción para los pensionados de Arquitectura.

(55) Ibídem. Art. 8. 
Saldrían después de Roma los pensionados para recorrer las ciudades de Italia y de Europa, pero no por eso cesaba la obligación de dependencia de los agentes que Su Majestad tenía en cada nación, ni la de seguir enviando dibujos y notas que atestiguasen su aprovechamiento. La instrucción especifica con todo detalle cómo deberían observar e informarse en cada ciudad acerca del modo y manera de estar trazadas las calles, edificados los templos, hospitales y monasterios, ideados y realizados los jardines, canales, presa, molinos y "demás artificios de agua». También los puertos y arsenales, los tribunales, universidades, fábricas, teatros y cuarteles entraban en el campo de observación y estudio de los arquitectos, quienes deberían escribir "cuanto alcancen y sepan de ellos, con expresión del temperamento, calidad del terreno, materiales, modo de emplearlos, etc., de suerte que no sólo ellos queden instruidos, sino también puedan instruir a otros con sus diseños y apuntaciones» (56).

En fin, el programa era tan ambicioso que podía resumirse en lo establecido en el artículo 20, donde se declaraba ser obligación de los pensionados "no dejar sin reconocer, dibujar y explicar por escrito objeto alguno de los muchos que dependen de la arquitectura y se hallan en los países mencionados». La Academia estaba persuadida de que por este camino los arquitectos enriquecerían notablemente sus ideas y seran útiles a su Patria.

No hay duda de que el Reglamento estaba amasado con buenos deseos y grandes ambiciones de superación. Pecaba de excesivo en todos los órdenes y así lo comprendieron los mismos académicos que lo habían elaborado y que al enviarlo al nuevo director hubieron de advertir que no pretendían que los pensionados hicieran todo lo que en él se preceptuaba, sino que se trataba de un plan máximo al que deberían tratar de aproximarse (57). Y esto fue la muerte del Reglamento, pues los pensionados de arquitectura (lo mismo que los de escultura y pintura) no se consideraron ya con la obligación de ajustarse a él, sino en una mínima parte. Y así quedó infructuoso el esfuerzo que los académicos habían hecho para su elaboración.

(56) Instrucción para los pensionados de Arquitectura. Art. 15 .

(57) En fecha 30 de noviembre de 1758. Leg. 49. Arch. Academia de San Fernando. 